\title{
One-Pot Synthesis of Cyclic Nitrones and Their Conversion to Pyrrolizidines: 7a-epi-Crotanecine Inhibits $\alpha$-Mannosidases
}

\author{
S. Cicchi, M. Marradi, P. Vogel, and A. Goti* \\ Dipartimento di Chimica Organica "Ugo Schiff”, Università di Firenze, ICCOM C.N.R., via della Lastruccia 13, \\ I-50019 Sesto Fiorentino (FI), Italy and Laboratory of Glycochemistry and Asymmetric Synthesis (LGSA), Ecole \\ Polytechnique Fédérale de Lausanne (EPFL), BCH, CH-1015 Lausanne, Switzerland \\ andrea.goti@unifi.it \\ Supporting Material
}

Table of contents

General

(2R,3S)-O-isopropylidene-4-O-tert-butyldimethylsilyl-D-erythraldoxime (15)

S2

(2R,3S)-O-isopropylidene-4-O-(tert-butyldimethylsilyl)-D-erythronitrile (16)

S2

1,3-Dipolar cycloaddition of $(3 S, 4 R)-3,4$-isopropylidenedioxypyrroline

1-oxide ((-)-3) with dimethyl maleate

S3

Reductive ring-opening of pyrroloisoxazolididines (-)-32 and (+)-33 to

pyrrolizidinones (-)-35 and (+)-36

Reduction of pyrrolizidinone (-)-35 to alcohol (+)-38

Synthesis of compound (+)-39

S5

Literature references

${ }^{1} \mathrm{H}$ NMR and ${ }^{13} \mathrm{C}$ NMR spectra of compound 5

${ }^{1}$ H NMR and ${ }^{13} \mathrm{C}$ NMR spectra of compound (-)-6

${ }^{1} \mathrm{H}$ NMR and ${ }^{13} \mathrm{C}$ NMR spectra of compound (-)-7·HCl

${ }^{1} \mathrm{H}$ NMR and ${ }^{13} \mathrm{C}$ NMR (APT) spectra of compound (-)-8· HCl 


\section{General}

All reactions requiring anhydrous conditions were carried out under nitrogen and solvents were appropriately dried before use. $R_{f}$ values refer to TLC on $0.25-\mathrm{mm}$ silica gel plates $\left(\right.$ Merck $\left.F_{254}\right)$ obtained using the same eluent as in the separation of the compound by flash column chromatography (FCC), except where indicated. Melting points (m.p.) were determined on a RCH Kofler apparatus and are uncorrected. ${ }^{1} \mathrm{H}$ and ${ }^{13} \mathrm{C}$ NMR spectra (in $\mathrm{CDCl}_{3}$ solution, unless otherwise stated) were recorded at $200 \mathrm{MHz}$ and 50.3 MHz, respectively; the chemical shift for ${ }^{1} \mathrm{H}$ and ${ }^{13} \mathrm{C}$ NMR spectra are given in ppm from TMS. Mass spectra (EI, $70 \mathrm{eV})$ were recorded by direct inlet.

(2R,3S)-O-isopropylidene-4-O-tert-butyldimethylsilyl-D-erythraldoxime (15). A solution of lactol (-)-9 (430 mg, $2.70 \mathrm{mmol})$ in dry pyridine $(5 \mathrm{~mL})$ was added with $3 \AA$ molecular sieves $(2.16 \mathrm{~g})$ and $\mathrm{NH}_{2} \mathrm{OH} \cdot \mathrm{HCl}(223 \mathrm{mg}, 3.24 \mathrm{mmol}, 1.2$ equiv). The mixture was stirred overnight at rt, then TBDMSCl (487 mg, $3.24 \mathrm{mmol}$ ) was added and the reaction mixture was stirred for $2 \mathrm{~d}$ at $\mathrm{rt}$. The suspension was diluted with dichloromethane $(5 \mathrm{~mL})$, filtered through a short pad of Celite and concentrated. The residue was purified by flash column chromatography (FCC) on silica gel to afford a mixture of oximes $15(140 \mathrm{mg}, 47 \%, Z: E=1: 8)$ as a white solid. $R_{f}=0.51$ (ethyl acetate:petroleum ether 1:3); mp 73-77 ${ }^{\circ} \mathrm{C} ;[\alpha]_{\mathrm{D}}{ }^{23}=-0.6\left(c 0.51, \mathrm{CH}_{2} \mathrm{Cl}_{2}\right) ;{ }^{1} \mathrm{H}$ NMR $\delta Z$ diastereoisomer: $7.10(\mathrm{bs}, 1 \mathrm{H}), 6.96(\mathrm{~d}, J=5.1 \mathrm{~Hz}$, 1H), $5.29(\mathrm{dd}, J=7.0,5.1 \mathrm{~Hz}, 1 \mathrm{H}), 4.48-4.41(\mathrm{~m}, 1 \mathrm{H}), 3.59-3.50(\mathrm{~m}, 2 \mathrm{H}), 1.52(\mathrm{~s}, 3 \mathrm{H}), 1.39(\mathrm{~s}, 3 \mathrm{H})$, $0.87(\mathrm{~s}, 9 \mathrm{H}), 0.05(\mathrm{~s}, 6 \mathrm{H}) ; E$ diastereoisomer: $7.64(\mathrm{bs}, 1 \mathrm{H}), 7.45(\mathrm{~d}, J=7.6 \mathrm{~Hz}, 1 \mathrm{H}), 4.71(\mathrm{t}, J=7.6$ $\mathrm{Hz}, 1 \mathrm{H}), 4.28(\mathrm{q}, J=7.6 \mathrm{~Hz}, 1 \mathrm{H}), 3.72-3.64(\mathrm{~m}, 2 \mathrm{H}), 1.49(\mathrm{~s}, 3 \mathrm{H}), 1.38(\mathrm{~s}, 3 \mathrm{H}), 0.88(\mathrm{~s}, 9 \mathrm{H}), 0.06$ (s, $6 \mathrm{H}) ;{ }^{13} \mathrm{C}$ NMR $\delta E$ diastereoisomer: $148.5(\mathrm{~s}), 109.5$ (s), $78.2(\mathrm{~d}), 74.9$ (d), $61.3(\mathrm{t}), 27.6(\mathrm{q}), 25.8$ (3C, q), $25.2(\mathrm{q}), 18.2(\mathrm{~s}),-5.5(2 \mathrm{C}, \mathrm{q})$; IR $\left(\mathrm{CHCl}_{3}\right) v_{\max } 3571,3058,2935,1210,1084 \mathrm{~cm}^{-1} ; \mathrm{MS} \mathrm{m} / \mathrm{z}(\%)$ 274 (4), 174 (30), 144 (46), 84 (58), 75 (100); Anal. calcd. for $\mathrm{C}_{13} \mathrm{H}_{27} \mathrm{NO}_{4} \mathrm{Si}: \mathrm{C}, 53.94 ; \mathrm{H}, 9.40 ; \mathrm{N}$, 4.84. Found: C, 53.90; H, 9.80; N, 5.15.

$(2 R, 3 S)-O$-isopropylidene-4- $O$-(tert-butyldimethylsilyl)-D-erythronitrile (16). A $0.2 \mathrm{M}$ solution of the oxime 15 in dry pyridine $(3 \mathrm{~mL})$ was cooled at $0{ }^{\circ} \mathrm{C}$ and added with $3 \AA$ molecular sieves $(240 \mathrm{mg})$ 
and 1.2 equiv. of cooled methanesulfonyl chloride at a $15 \mu \mathrm{L} / \mathrm{min}$ rate. The reaction was stirred at $0{ }^{\circ} \mathrm{C}$ for $2 \mathrm{~h}$ and then left stirring overnight at $\mathrm{rt}$. The mixture was diluted in dichloromethane $(3 \mathrm{~mL})$, filtered through a Celite pad, and concentrated. The residue was purified by FCC on silica gel to afford $\mathbf{1 6}$ (51 $\mathrm{mg}, 31 \%$ ) as a colorless oil. $R_{f}=0.51$ (eluent ethyl acetate:petroleum ether $\left.1: 5\right) ;{ }^{1} \mathrm{H}$ NMR $\delta 4.85(\mathrm{~d}, J=$ $5.4 \mathrm{~Hz}, 1 \mathrm{H}), 4.34-4.25(\mathrm{~m}, 1 \mathrm{H}), 4.09-3.89(\mathrm{~m}, 1 \mathrm{H}), 4.06-3.84(\mathrm{~m}, 1 \mathrm{H}), 1.55(\mathrm{~s}, 3 \mathrm{H}), 1.38(\mathrm{~s}, 3 \mathrm{H}), 0.90$ (s, 9H), $0.10(\mathrm{~s}, 6 \mathrm{H}) ;{ }^{13} \mathrm{C}$ NMR $\delta 116.3(\mathrm{~s}), 112.6(\mathrm{~s}), 77.2(\mathrm{~d}), 67.5(\mathrm{~d}), 62.0(\mathrm{t}), 27.0(\mathrm{q}), 25.8(\mathrm{q})$, 25.7 (3C, q), $18.2(\mathrm{~s}),-5.5(2 \mathrm{C}, \mathrm{s})$; IR $\left(\mathrm{CHCl}_{3}\right) v_{\max } 3693,2958,2933,2253,1599,1376,1215,1089$ $\mathrm{cm}^{-1}$; MS m/z (\%) 191 (3), 167 (10), 149 (45), 84 (66), 71 (61), 69 (50), 57 (100); Anal. calcd. for $\mathrm{C}_{13} \mathrm{H}_{25} \mathrm{NO}_{3} \mathrm{Si}: \mathrm{C}, 57.53 ; \mathrm{H}, 9.28 ; \mathrm{N}, 5.16$. Found: C, 57.27; H, 9.01; N, 5.53.

1,3-Dipolar cycloaddition of (3S,4R)-3,4-isopropylidenedioxypyrroline 1-oxide ((-)-3) with dimethyl maleate $^{\mathrm{S} 1}$

Dimethyl maleate (2 equiv) was added to a $0.5 \mathrm{M}$ solution of the enantiopure nitrone (-)-3 (1 mmol) in dichloromethane. The mixture was stirred at $\mathrm{rt}$ for $15 \mathrm{~h}$ and then concentrated to give a 9.6:6:1 mixture (by ${ }^{1} \mathrm{H}$ NMR integration) of cycloadducts (-)-32, (+)-33 and (-)-34 with a $92 \%$ total yield. Purification by FCC (eluent ethyl acetate:petroleum ether 1:1) afforded the enantiopure adducts (+)-33 $\left(R_{f}=0.45,33 \%\right)$ and $(-)-32\left(R_{f}=0.33,53 \%\right)$, whose spectroscopic data were in agreement with those reported in the literature for the racemates, ${ }^{\mathrm{S} 1}$ and $(-)-\mathbf{3 4}\left(R_{f}=0.13,6 \%\right)$.

(-)-32. Colorless oil. $[\alpha]_{D}^{21}-10\left(c 0.95, \mathrm{CHCl}_{3}\right)$. Anal. calcd. for $\mathrm{C}_{13} \mathrm{H}_{19} \mathrm{NO}_{7}: \mathrm{C}, 51.82 ; \mathrm{H}, 6.36 ; \mathrm{N}$, 4.65. Found: C, 51.89; H, 6.23; N, 4.60.

(+)-33. White solid. Mp 80-81 ${ }^{\circ} \mathrm{C}$; $[\alpha]_{\mathrm{D}}{ }^{21}+75\left(\right.$ c $\left.1.43, \mathrm{CH}_{2} \mathrm{Cl}_{2}\right)$. Anal. calcd. for $\mathrm{C}_{13} \mathrm{H}_{19} \mathrm{NO}_{7}$ : $\mathrm{C}$, 51.82; H, 6.36; N, 4.65. Found: C, 52.00; H, 6.41; N, 4.73.

(-)-34. Colorless oil. $[\alpha]_{\mathrm{D}}{ }^{20}-79\left(c 0.30, \mathrm{CHCl}_{3}\right) ;{ }^{1} \mathrm{H}$ NMR $\delta 4.88(\mathrm{t}, J=5.4 \mathrm{~Hz}, 1 \mathrm{H}), 4.84(\mathrm{~d}, J=9.0$ $\mathrm{Hz}, 1 \mathrm{H}), 4.72(\mathrm{t}, J=6.0 \mathrm{~Hz}, 1 \mathrm{H}), 4.20(\mathrm{dd}, J=9.2,2.4 \mathrm{~Hz}, 1 \mathrm{H}), 3.97(\mathrm{dd}, J=5.4,2.2 \mathrm{~Hz}, 1 \mathrm{H}), 3.75(\mathrm{~d}$, $J=15.9 \mathrm{~Hz}, 1 \mathrm{H}), 3.75(\mathrm{~s}, 3 \mathrm{H}), 3.71(\mathrm{~s}, 3 \mathrm{H}), 3.17(\mathrm{dd}, J=15.9,5.3 \mathrm{~Hz}, 1 \mathrm{H}), 1.46(\mathrm{~s}, 3 \mathrm{H}), 1.31(\mathrm{~s}, 3 \mathrm{H})$;

${ }^{13} \mathrm{C}$ NMR $\delta 170.7(\mathrm{~s}), 170.5(\mathrm{~s}), 113.3(\mathrm{~s}), 83.2$ (d), 81.2 (d), 78.1 (d), 71.4 (d), $59.4(\mathrm{t}), 52.4(\mathrm{q}, 2 \mathrm{C})$, 
51.1 (d), 26.4 (q), 24.1 (q); IR $\left(\mathrm{CHCl}_{3}\right) v_{\max }$ 2993, 2955, 2937, 1740, 1437, 1372, 1243, 1206, 1082 $\mathrm{cm}^{-1}$; MS m/z (\%) $301\left(\mathrm{M}^{+}, 47\right), 286$ (4), 198 (30), 82 (61), 59 (100). Anal. calcd. for $\mathrm{C}_{13} \mathrm{H}_{19} \mathrm{NO}_{7}: \mathrm{C}_{\text {, }}$ 51.82; H, 6.36; N, 4.65. Found: C, 51.80; H, 6.76; N, 4.95.

\section{Reductive ring-opening of pyrroloisoxazolididines (-)-32 and (+)-33 to pyrrolizidinones (-)-35} and (+)-36

A solution of the adduct (-)-32 or (+)-33 $(1 \mathrm{mmol})$ in methanol $(10 \mathrm{~mL})$ was added with $\mathrm{Pd}(\mathrm{OH})_{2} / \mathrm{C}$ $(10 \% \mathrm{Pd}, 150 \mathrm{mg})$ and stirred overnight under $\mathrm{H}_{2}$ atmosphere at $\mathrm{rt}$. The suspension was then filtered through a short pad of Celite, concentrated and purified by FCC.

(-)-35. White solid (247 mg, 91\%). $R_{f}=0.20$ (eluent ethyl acetate:petroleum ether 5:1); mp 160-162 ${ }^{\circ} \mathrm{C} ;[\alpha]_{\mathrm{D}}{ }^{20}-76\left(c 1.01, \mathrm{CHCl}_{3}\right)$. Spectroscopic data are in agreement with those reported. ${ }^{\mathrm{S} 1}$

(+)-36. White solid (130 mg, 48\%). $R_{f}=0.32$ (eluent ethyl acetate:petroleum ether 5:1); mp 104-106 ${ }^{\circ} \mathrm{C} ;[\alpha]_{\mathrm{D}}{ }^{20}+35\left(c 0.58, \mathrm{CHCl}_{3}\right) ;{ }^{1} \mathrm{H}$ NMR $\delta 4.79(\mathrm{td}, J=6.6,3.2 \mathrm{~Hz}, 1 \mathrm{H}), 4.55(\mathrm{dd}, J=5.6,3.0 \mathrm{~Hz}$, $1 \mathrm{H}), 4.28(\mathrm{dd}, J=6.6,6.2 \mathrm{~Hz}, 1 \mathrm{H}), 4.12(\mathrm{dd}, J=12.8,6.6 \mathrm{~Hz}, 1 \mathrm{H}), 4.11(\mathrm{dd}, J=8.5,6.2 \mathrm{~Hz}, 1 \mathrm{H}), 3.79$ (s, 3H), $3.44(\mathrm{dd}, J=8.5,5.6 \mathrm{~Hz}, 1 \mathrm{H}), 3.31(\mathrm{~d}, J=3.0 \mathrm{~Hz}$, exchanging proton, $1 \mathrm{H}), 3.20(\mathrm{dd}, J=12.8$, $3.2 \mathrm{~Hz}, 1 \mathrm{H}), 1.57$ (s, 3H), $1.31(\mathrm{~s}, 3 \mathrm{H}) ;{ }^{13} \mathrm{C} \mathrm{NMR} \delta 171.3(\mathrm{~s}), 170.7$ (s), 114.8 (s), 80.5 (d), 79.5 (d), $73.8(\mathrm{~d}), 64.3(\mathrm{~d}), 52.5(\mathrm{~d}), 48.1(\mathrm{q}), 47.0(\mathrm{t}), 27.5(\mathrm{q}), 25.3(\mathrm{q}) ; \mathrm{IR}(\mathrm{KBr}) v_{\max } 3592,2987,2956,1736$, 1702, 1438, 1373, 1342, $1210 \mathrm{~cm}^{-1}$; MS m/z (\%) $271\left(\mathrm{M}^{+}, 2\right), 256(33), 196(32), 156(100), 136(62)$, 126 (40), 98 (53), 96 (62), 84 (61), 59 (51). Anal. calcd. for $\mathrm{C}_{12} \mathrm{H}_{17} \mathrm{NO}_{6}$ : C, 53.13; H, 6.32; N, 5.16. Found: C, 53.08; H, 6.36; N, 5.14.

\section{Reduction of pyrrolizidinone (-)-35 to alcohol (+)-38}

$\mathrm{BH}_{3} \mathrm{SMe}_{2}$ (ca. $10 \mathrm{M}$ in $\mathrm{BH}_{3}, 0.2 \mathrm{~mL}, 2$ equiv) was added dropwise to a suspension of the pyrrolizidinone (-)-35 $(271 \mathrm{mg}, 1 \mathrm{mmol})$ in dry THF $(10 \mathrm{~mL})$ cooled to $0{ }^{\circ} \mathrm{C}$. After complete dissolution of the reagent, the mixture was heated at reflux for $1 \mathrm{~h}$ and then cooled to $0{ }^{\circ} \mathrm{C}$. The reaction was quenched by dropwise addition of an excess (6 equiv with respect to $\mathrm{BH}_{3}$ ) of EtOH, followed by a $0.25 \mathrm{M} \mathrm{NaOH}$ aq. solution. Water $(10 \mathrm{~mL})$ was then added and the product was extracted with ethyl 
acetate $(2 \times 10 \mathrm{~mL})$. The collected organic phase was dried over $\mathrm{Na}_{2} \mathrm{SO}_{4}$, filtered and concentrated in vacuo. The residue was purified by column chromatography affording compound (+)-38 as a hygroscopic white solid $(210 \mathrm{mg}, 81 \%) . R_{f}=0.56$ (ethyl acetate:petroleum ether 5:2); $\mathrm{mp} 91-92{ }^{\circ} \mathrm{C}$; $[\alpha]_{\mathrm{D}}{ }^{25}+14\left(c 0.43, \mathrm{CH}_{2} \mathrm{Cl}_{2}\right) ;{ }^{1} \mathrm{H}$ NMR $\delta 5.04-4.89(\mathrm{~m}, 2 \mathrm{H}), 4.75(\mathrm{dd}, J=5.9,2.9 \mathrm{~Hz}, 1 \mathrm{H}), 4.24(\mathrm{dd}, J=$ 5.9, $2.2 \mathrm{~Hz}, 1 \mathrm{H}), 3.79(\mathrm{~s}, 3 \mathrm{H}), 3.66-3.45(\mathrm{~m}, 3 \mathrm{H}), 3.00-2.89(\mathrm{~m}, 2 \mathrm{H}), 1.60(\mathrm{~s}, 3 \mathrm{H}), 1.32(\mathrm{~s}, 3 \mathrm{H}) ;{ }^{13} \mathrm{C}$ NMR $\delta 169.2(s), 113.8(s), 86.4(d), 78.8(d), 78.1(d), 72.7(d), 69.4(t), 68.2(t), 56.3(d), 52.7(q)$, 26.7 (q), 25.0 (q); IR (KBr) $v_{\max } 3422,2988,2940,2368,2327,2277,1735,1716,1438,1383,1213$ $\mathrm{cm}^{-1} ; \mathrm{MS} \mathrm{m} / \mathrm{z}(\%) 257\left(\mathrm{M}^{+}, 13\right), 256\left(\mathrm{M}^{+}-1,13\right), 183(45), 181$ (93), $168(45), 157(65), 155(89), 138$ (51), 120 (56), 96 (100), 84 (51), 79 (77). Anal. calcd. for $\mathrm{C}_{12} \mathrm{H}_{19} \mathrm{NO}_{5} \cdot \mathrm{H}_{2} \mathrm{O}: \mathrm{C}, 52.35 ; \mathrm{H}, 7.69 ; \mathrm{N}, 5.09$. Found: C, 52.62; H, 8.01; N, 5.12.

\section{Synthesis of compound (+)-39}

A solution of compound (+)-38 (200 mg, $0.7 \mathrm{mmol})$ and triethylamine (1.5 equiv) in $\mathrm{CH}_{2} \mathrm{Cl}_{2}(7 \mathrm{~mL})$ was cooled in an ice bath and added with methanesulfonyl chloride (133 mg, $1.17 \mathrm{mmol}, 1.5$ equiv). The reaction was stirred at $\mathrm{rt}$ for $15 \mathrm{~h}$. DBU (266 mg, $1.75 \mathrm{mmol})$ was then added and the mixture was stirred for $30 \mathrm{~min}$. The solution was diluted with dichloromethane $(20 \mathrm{~mL})$ and washed with water $(3 \times 20 \mathrm{~mL})$, saturated $\mathrm{NaHCO}_{3}(20 \mathrm{~mL})$ and brine $(20 \mathrm{~mL})$. The organic phase was dried over $\mathrm{Na}_{2} \mathrm{SO}_{4}$, filtered, concentrated in vacuo and the crude residue was purified by column chromatography to afford compound (+)-39 as a hygroscopic white solid (135 mg, 81\%). $R_{f}=0.42$ (dichloromethane); mp 85-86 ${ }^{\circ} \mathrm{C} ;[\alpha]_{\mathrm{D}}{ }^{24}+22\left(c=0.08, \mathrm{CH}_{3} \mathrm{OH}\right) ;{ }^{1} \mathrm{H}$ NMR $\delta 6.66(\mathrm{q}, J=2.2 \mathrm{~Hz}, 1 \mathrm{H}), 4.77-4.68(\mathrm{~m}, 3 \mathrm{H}), 4.25(\mathrm{dt}, J$ $=17.5,2.2 \mathrm{~Hz}, 1 \mathrm{H}), 3.86-3.74(\mathrm{~m}, 1 \mathrm{H}), 3.83(\mathrm{~s}, 3 \mathrm{H}), 3.56(\mathrm{dd}, J=12.5,5.1 \mathrm{~Hz}, 1 \mathrm{H}), 3.32(\mathrm{dd}, J=$ 12.5, 5.9 Hz, 1H), $1.61(\mathrm{~s}, 3 \mathrm{H}), 1.32(\mathrm{~s}, 3 \mathrm{H}) ;{ }^{13} \mathrm{C}$ NMR $\delta 169.2(\mathrm{~s}), 136.6(\mathrm{~d}), 132.0(\mathrm{~s}), 113.7$ (s), 83.1 (d), 82.7 (d), $78.4(\mathrm{~d}), 70.9$ (t), 67.5 (t), 52.3 (q), 26.8 (q), 25.2 (q); IR (KBr) $v_{\max }$ 2990, 2956, 2380, 2323, 2275, 1738, 1718, 1661, 1458, 1434, 1385, 1373, $1272 \mathrm{~cm}^{-1} ; \mathrm{MS}\left(\mathrm{CDCl}_{3}\right) \mathrm{m} / \mathrm{z}(\%) 239\left(\mathrm{M}^{+}, 3\right)$, 224 (3), 86 (55), 84 (100), 56 (83). Anal. calcd. for $\mathrm{C}_{12} \mathrm{H}_{17} \mathrm{NO}_{4} \mathrm{H}_{2} \mathrm{O}$ : C, 56.02; H, 7.44; N, 5.44. Found: C, 56.40; H, 7.79; N, 5.54. 
(S1) Goti, A.; Cicchi, S.; Cacciarini, M.; Cardona, F.; Fedi, V.; Brandi, A. Eur. J. Org. Chem. 2000, 3633-3645. 
mm38tris fr142 nitrone Boc

Pulse Sequence: s2pul

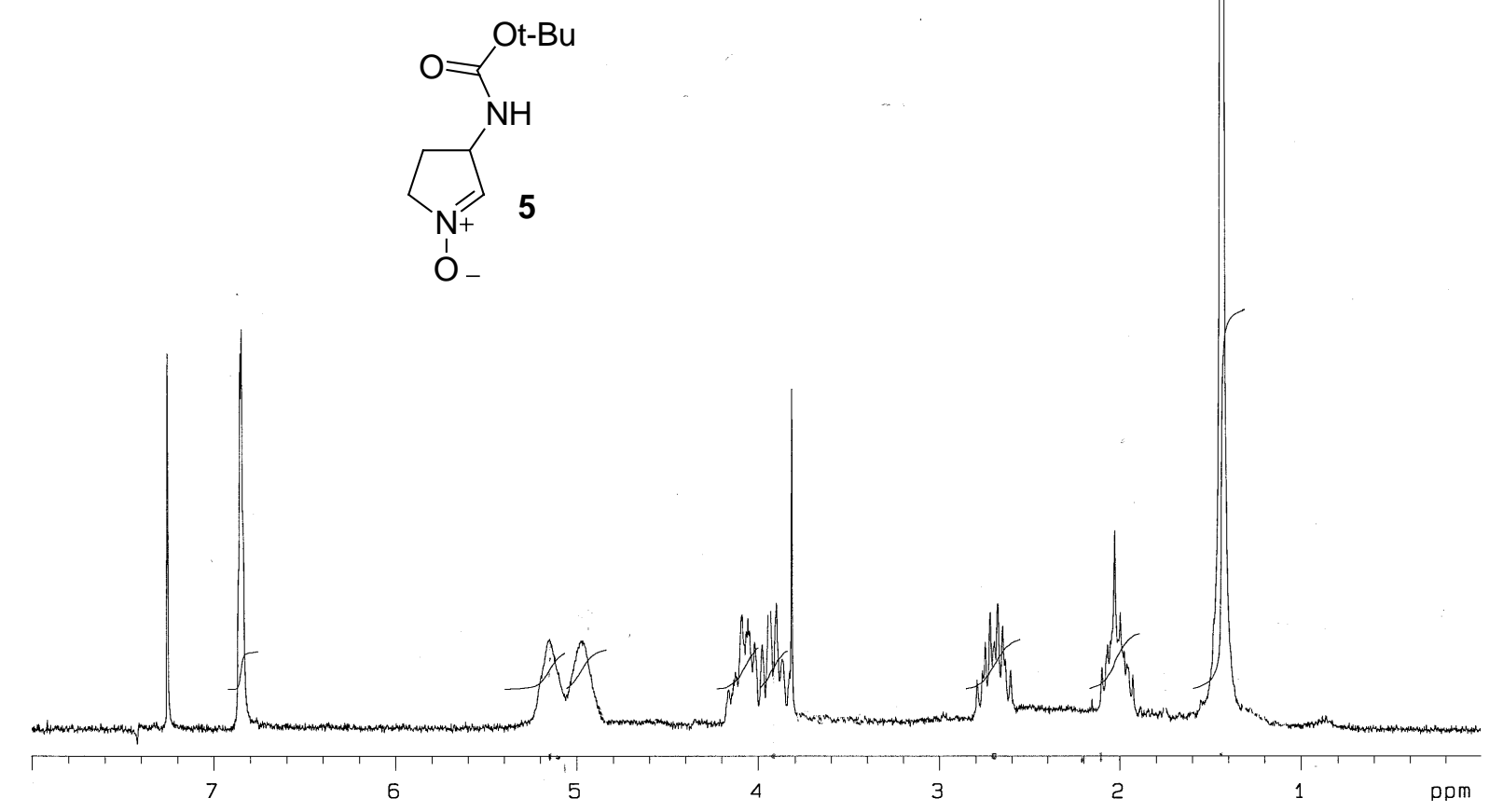

13C OBSERVE

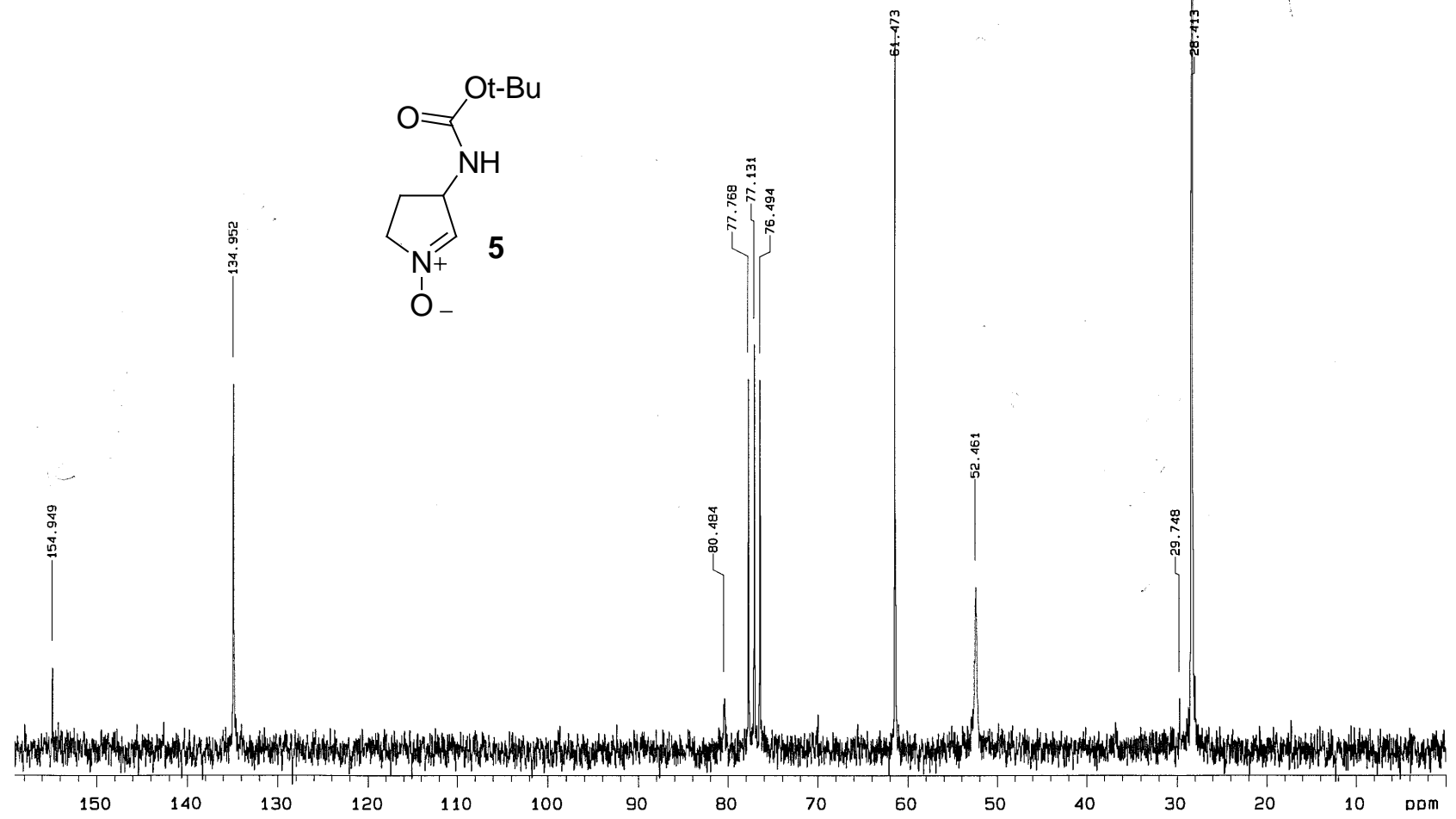




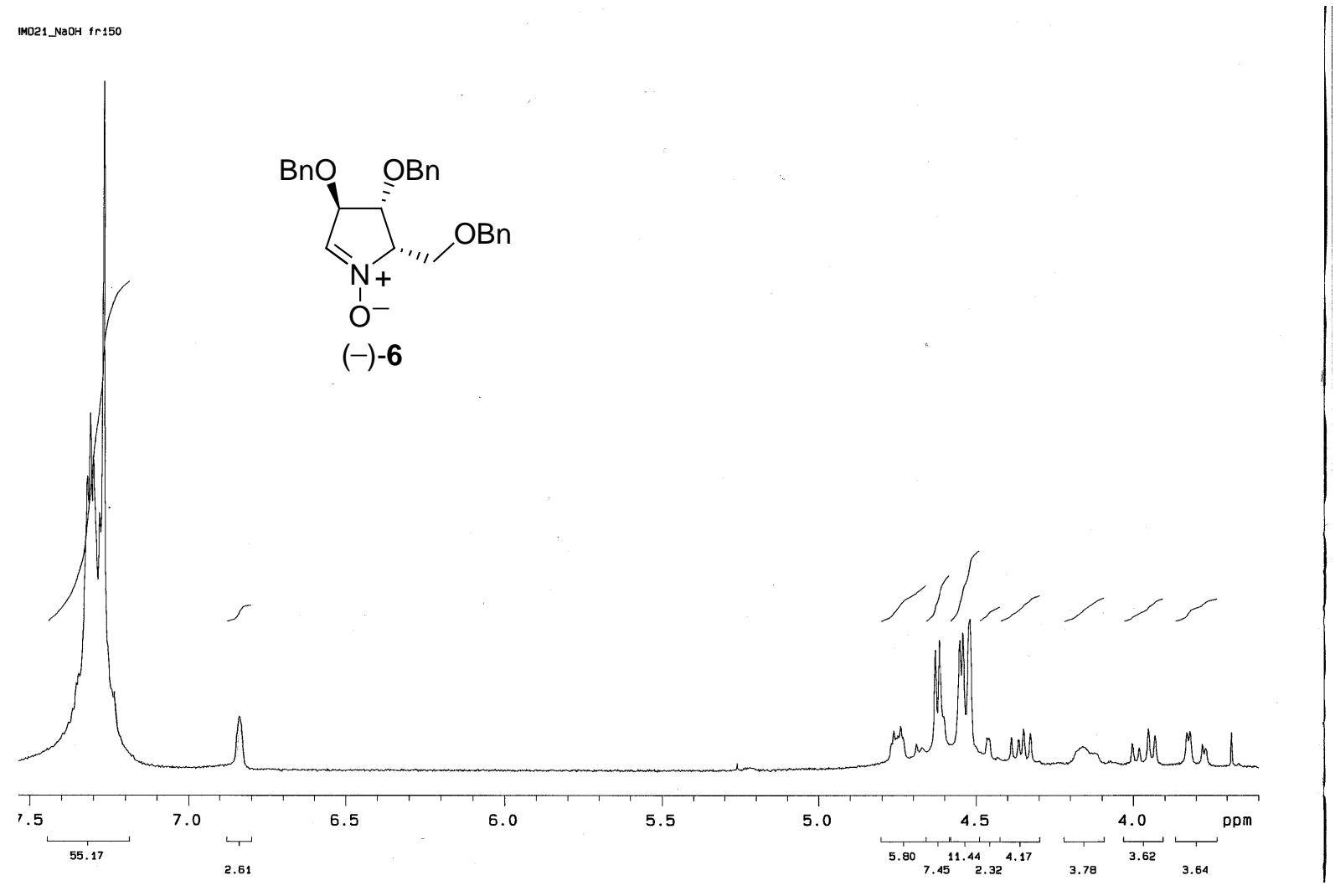

MMD21_NaOH Fr150

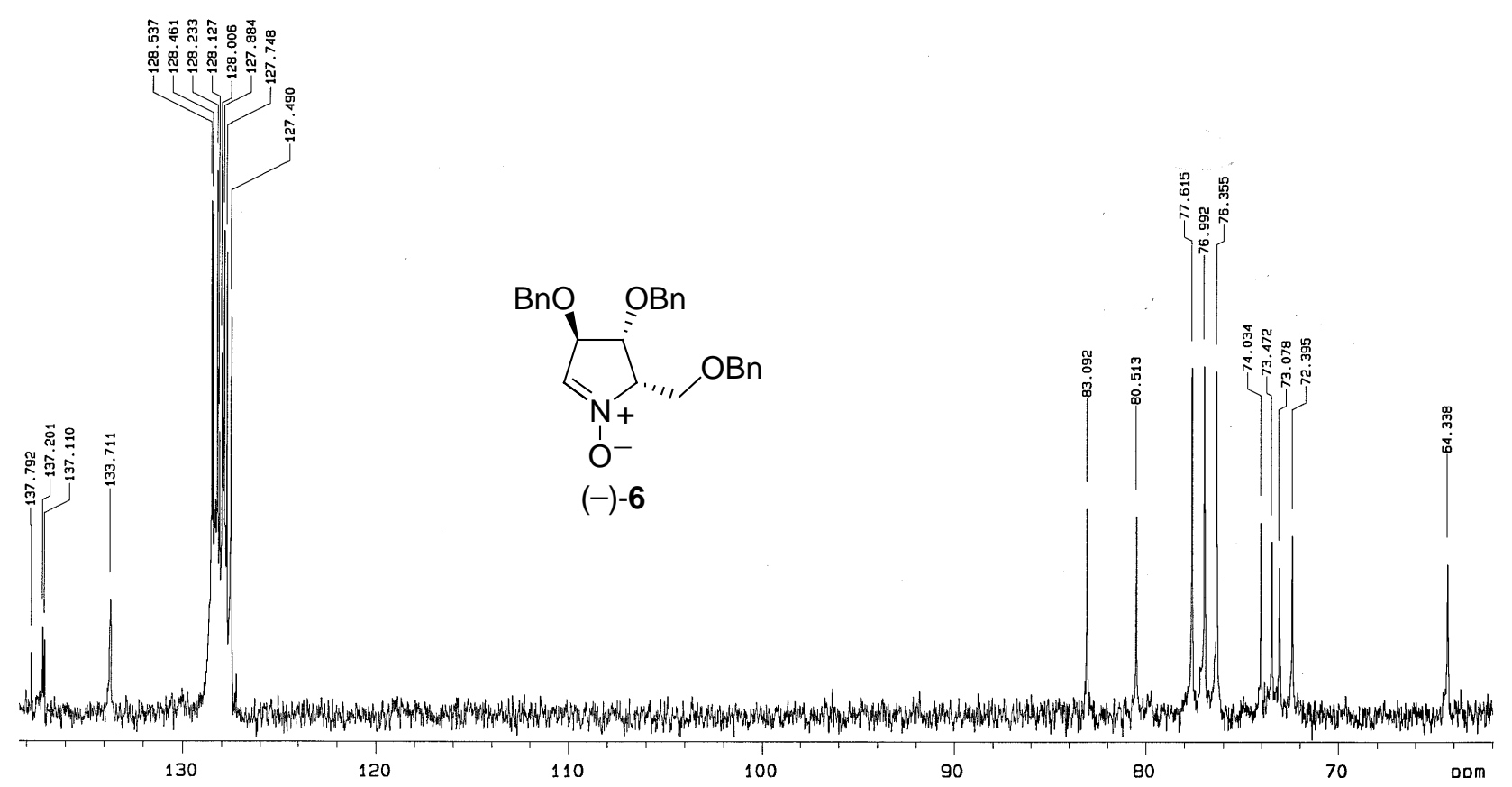




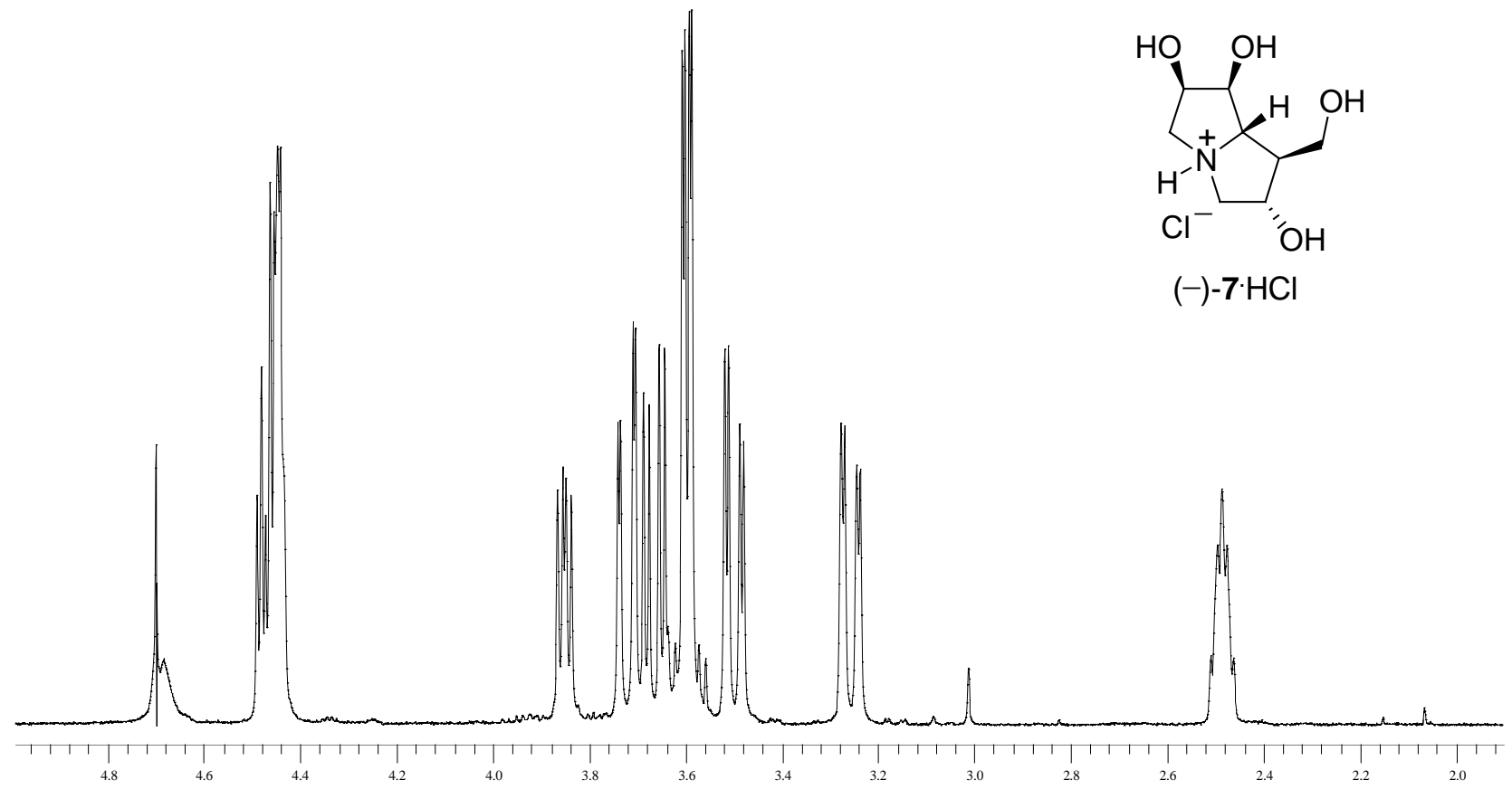

MMlOS 3

135 in 020

HCl $1 M$ in MeOH per deprotez. Dírrolizidinone rldotto
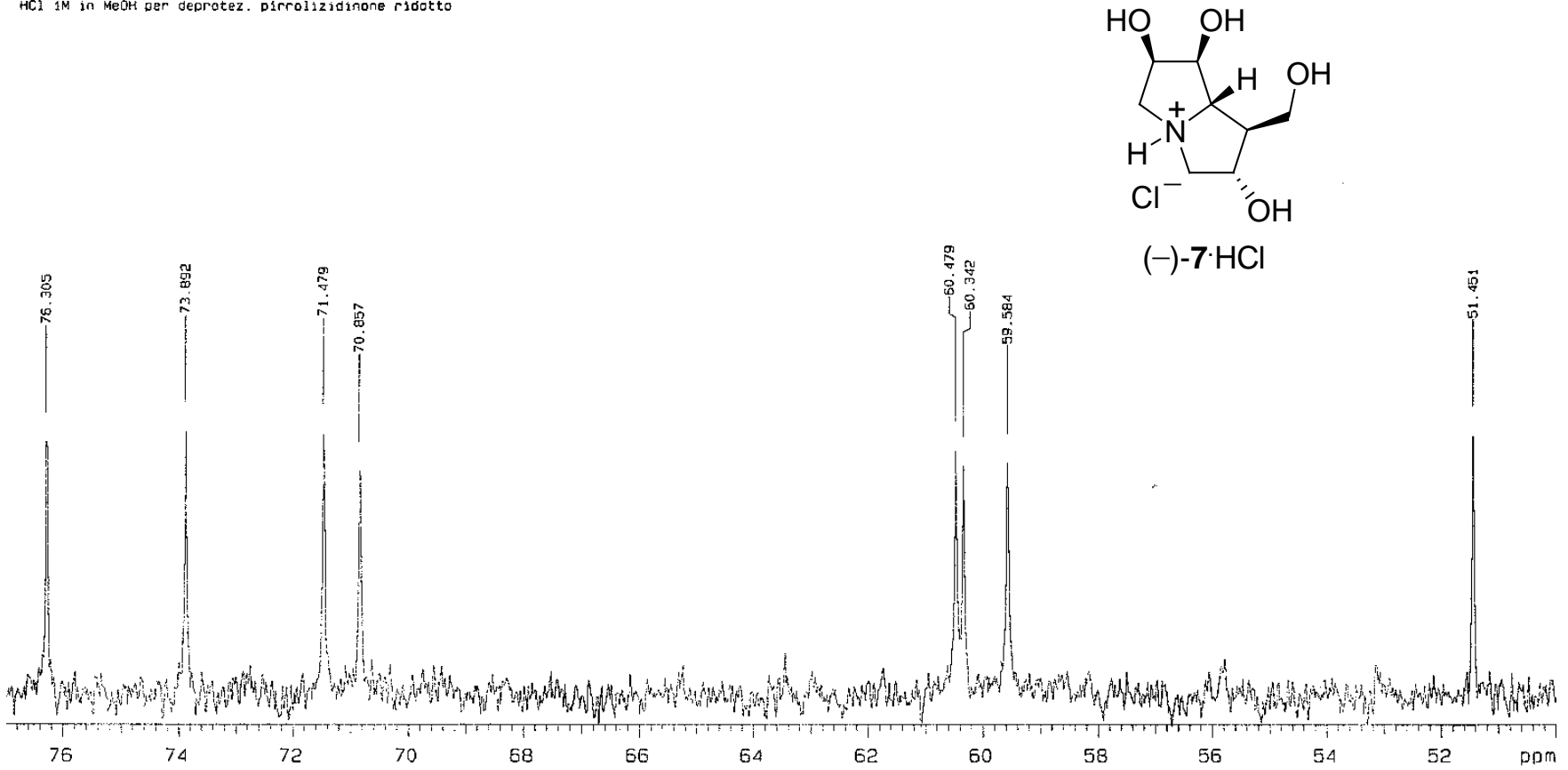

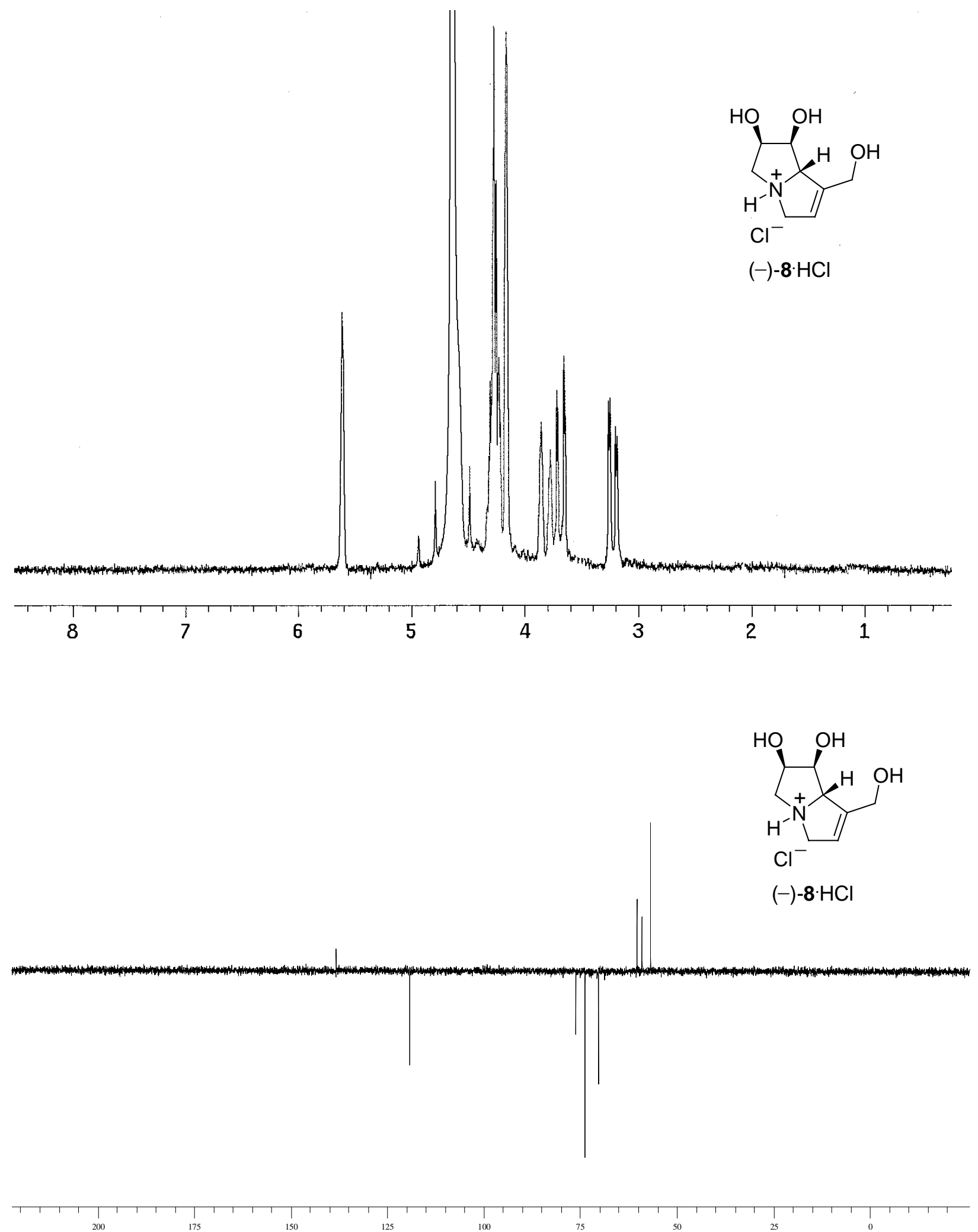